\title{
Gene- and Genome-Based Analysis of Significant Codon Patterns in Yeast, Rat and Mice Genomes with the CUT Codon Utilization Tool
}

Frank Doyle ${ }^{1^{*}}$, Andrea Leonardi $i^{*}$, Lauren Endres ${ }^{2 *}$, Scott A. Tenenbaum ${ }^{1}$, Peter C. Dedon ${ }^{3,4}$ and Thomas J. Begley ${ }^{1,5 \#}$

${ }^{1}$ State University of New York - SUNY Polytechnic Institute, College of Nanoscale Science and Engineering, Albany, NY; ${ }^{2}$ State University of New York - SUNY Polytechnic Institute, College of Arts and Sciences, Utica, NY; ${ }^{3}$ Department of Biological Engineering and Center for Environmental Health Science, Massachusetts Institute of Technology, Cambridge, MA; ${ }^{3}$ Singapore-MIT Alliance for Research and Technology, Singapore; ${ }^{5}$ RNA Institute, University at Albany, State University of New York.

${ }^{*}$ Authors contributed equally

Keywords: anticodon, codon bias, gene expression, modification tunable transcripts, stress response, translation, RNA modification. 


\section{Abstract}

The translation of mRNA in all forms of life uses a three-nucleotide codon and aminoacyl-tRNAs to synthesize a protein. There are 64 possible codons in the genetic code, with codons for the $\sim 20$ amino acids and 3 stop codons having 1- to 6-fold degeneracy. Recent studies have shown that families of stress response transcripts, termed modification tunable transcripts (MoTTs), use distinct codon biases that match specifically modified tRNAs to regulate their translation during a stress. Similarly, translational reprogramming of the UGA stop codon to generate selenoproteins or to perform programmed translational read-through (PTR) that results in a longer protein, requires distinct codon bias (i.e., more than one stop codon) and, in the case of selenoproteins, a specifically modified tRNA. In an effort to identify transcripts that have codon usage patterns that could be subject to translational control mechanisms, we have used existing genome and transcript data to develop the gene-specific Codon UTilization (CUT) tool and database, which details all 1-, 2-, 3-, 4- and 5codon combinations for all genes or transcripts in yeast (Saccharomyces cerevisiae), mice (Mus musculus) and rats (Rattus norvegicus). Here, we describe the use of the CUT tool and database to characterize significant codon usage patterns in specific genes and groups of genes. In yeast, we demonstrate how the CUT database can be used to identify genes that have runs of specific codons (e.g., AGA, GAA, AAG) linked to translational regulation by tRNA methyltransferase 9 (Trm9). We further demonstrate how groups of genes can be analyzed to find significant dicodon patterns, with the 80 Gcn4-regulated transcripts significantly $(P<$ 0.00001) over-represented with the AGA-GAA dicodon. We have also used the CUT database to identify mouse and rat transcripts with internal UGA codons, with the surprising finding of 45 and 120 such transcripts, respectively, which is much larger than expected. The UGA data suggest that there could be many more translationally reprogrammed transcripts than currently reported. CUT thus represents a multi-species codoncounting database that can be used with mRNA-, translation- and proteomics-based results to better understand and model translational control mechanisms. 


\section{Introduction}

Translation is the process by which proteins are synthesized from messenger RNA (mRNA) in the ribosome, using both transfer RNAs (tRNAs) and other trans-acting factors. The codons in mRNA consist of three nucleotides and interact with the tRNA anticodon during translation to specify the insertion of one of $\sim 20$ amino acids in a growing peptide chain, or in the case of stop codons, to terminate translation. tRNA molecules consist of 70 to 90 nucleotides folded into a cloverleaf structure containing an anticodon loop and a 3'-CAA that is covalently linked to a specific amino acid. tRNA is initially transcribed to contain adenosine (A), guanosine $(\mathrm{G})$, uridine $(\mathrm{U})$ and cytidine $(\mathrm{C})$, but it is the most heavily modified nucleic acid in the cell and contains an average of 9-11 post-transcriptional modifications at $>30$ conserved sites [1, 2]. These RNA modifications have been shown play important roles in promoting tRNA stability, optimizing codon-anticodon interactions and preventing translation errors [3, 4].

In mRNA there are 64 possible three-nucleotide combinations that comprise the full set of codons. AUG is generally used as the start codon and the translation initiation site, but UUG, GUG and CUG have also been identified as occasional start sites in some transcripts in several organisms [5]. The ribosome has three sites associated with bound tRNA: one where the aminoacyl-tRNA enters ( $A$ site), one that has the tRNA bound to the growing polypeptide chain (P site), and lastly an E site where tRNA exits the ribosome. The polypeptide chain is catalytically transferred from the tRNA at the P site to the tRNA at the A site, at which point the free tRNA exits through the $E$ site and the ribosome moves along to the next codon [6]. Excluding stop codons, there are 61 possible codons that can occupy each site or more than 226,918 three-codon combinations that can associate with A-, P- and E-sites. Translation elongation continues until the ribosome encounters one of three stop codons, UAA, UAG or UGA. There are instances, described below, where a UGA stop codon, in conjunction with trans- and cis-acting factors, can signal for the addition of a unique amino acid and these instances highlight the use of codon-based translational regulatory mechanisms.

The regulation of gene expression by specific patterns of codon usage has been established for both prokaryotic and eukaryotic systems [7-12]. In bacterial systems where transcription is coupled to translation, the expression of tryptophan metabolic enzymes from the Trp operon is linked to the translation of the UGGUGG dicodon, where UGG codes for tryptophan, in a short leader peptide. The transcription and subsequent 
translation of tryptophan metabolic enzymes requires pausing at the UGG-UGG dicodon due to low levels of charged tryptophan tRNA, which promotes specific folding of the upstream transcript (the attenuator) to promote the transcription of the entire Trp operon [7]. Translational regulation has also been demonstrated in budding yeast for some stress response genes over-represented with codons linked to leucine, arginine and glutamic acid, by the regulated levels of modified cytidines and uridines in the wobble position of the tRNA anticodon. tRNA methyltransferase (Trm) 4 and $\operatorname{Trm} 9$ complete the formation of 5-methylcytidine $\left(\mathrm{m}^{5} \mathrm{C}\right)$ and 5-methoxycarbonylmethyluridine $\left(\mathrm{mcm}^{5} \mathrm{U}\right)$ and 5-methoxycarbonylmethyl-2-thiouridine $\left(\mathrm{mcm}^{5} \mathrm{~s}^{2} \mathrm{U}\right)$, respectively, in the anticodon of tRNAs specific for Leu, Arg and Glu $[8,9,13]$, Further, the levels of $\mathrm{m}^{5} \mathrm{C}$ $\left(\mathrm{H}_{2} \mathrm{O}_{2}, \mathrm{ROS}\right.$ agents), $\mathrm{mcm}{ }^{5} \mathrm{U}\left(\mathrm{HU}, \mathrm{MMS}\right.$, alkylating agents) and $\mathrm{mcm}^{5} \mathrm{~s}^{2} \mathrm{U}$ (HU, MMS, alkylating agents) modifications have been shown to change in response to stress $[8,9,14,15]$. Reporter, targeted, and systems-based studies comparing transcripts and proteins have demonstrated that the translation of UUG (Leu), AGA (Arg) and GAA (Glu) codons are dependent on specific wobble base modifications and that some stress response genes that over-use these codons have decreased translation in the absence of the corresponding Trm and wobble base modification $[9,10,14,16]$.

Links between the regulation of translation and distinct codon usage patterns has also been demonstrated for mammalian transcripts that use UGA stop codon reprogramming or programmed translational read-through (PTR). Stop codon recoding is used to incorporate selenocysteine into selenoproteins, with the corresponding activities involved in ROS detoxification, selenium utilization and thyroid function $[12,17]$. Selenocysteine has been referred to as the 21 st amino acid, with no dedicated triplet codon $[18,19]$. As such, decoding for selenocysteine is unconventional and requires a "recoding" of the UGA stop codon for incorporation in a process termed stop codon reprogramming $[17,19,20]$. Similar to the yeast Trm9 example noted earlier, stop codon recoding utilizes specifically modified wobble uridine bases, $\mathrm{mcm}^{5} \mathrm{U}$ and 5 methoxycarbonylmethyl-2'-O-methyluridine $\left(\mathrm{mcm}^{5} \mathrm{Um}\right)$, to promote optimal anticodon-codon interactions [17, 21, 22], as well as 3'-UTR regulatory sequence and trans-acting factors. The 3'-UTR found in transcripts encoding selenoproteins contains a selenocysteine insertion sequence (SECIS) that helps identify the internal UGA codons being re-coded for selenocysteine [23, 24]. PTR has also been shown to occur at UGA stop codons, as in the recent notable example of human vascular endothelial growth factor-A (VEGFA) isoform VEGF-Ax, in which Ax denotes an extended form [25]. VEGFA encodes a growth factor that promotes 
endothelial cell migration and growth, with roles in angiogenesis and tumorigenesis, among others [26]. VEGFA contains another stop codon that is 22 codons (66 nts) down from the first UGA stop codon, with the nucleotide sequence well conserved in humans, rats and mice. PTR of the first UGA stop codon inserts serine and promotes the formation of a 22 amino acid extended version (VEGF-Ax), with PTR requiring a cis-acting element found in the Ax region that is $63 \mathrm{nts}$ downstream of the canonical stop codon and corresponds to an hnRNP1 A2/B1 element [25]. With similarities to stop codon recoding, PTR uses cis-acting sequences, but the precise tRNA involved in PTR has not been clearly identified. PTR has also been shown for Ago1 and Mtch2 mRNA, and is predicted for Nr1d1, Adamts4 and Tox [25]. Other examples of translational read-through (Mdh1 and LdhB) have been reported to include stop codons outside of UGA, with targeted and genome-wide approaches identifying real and potential targets in humans, fruit flies, yeast and viruses [27].

Codon usage is one of the many parameters involved in the translational regulation of specific transcripts. Monocodon usage data is readily available to describe genome-based trends [28] and, in the case of yeast, to describe individual genes [13]. Bacterial studies on the Trp and other operons for other metabolic processes have demonstrated that dicodons in individual genes can have regulatory effects [29]. To investigate the potential regulatory effects of gene-specific dicodons and other codon combinations, we have developed a Codon UTilization (CUT) tool and database to analyze all genes or transcripts banked for a species, and we applied this tool to the analysis of yeast, mouse and rat genome sequence data (http://pare.sunycnse.com/cut/index.jsp). We demonstrate in yeast that specific dicodon and quadruple codon runs can be linked to optimal translation by Trm9-catalyzed tRNA. Further, we show that the AGA-GAA dicodon is over-represented in 80 Gcn4-regulated transcripts, which provides a novel link between the regulation of translation initiation in the Gcn2-elF2 $\alpha-G c n 4$ pathway and regulation of translational elongation by Trm9. Finally, we demonstrate how CUT can be used as an exploratory tool in identifying 45 mouse and 120 rat transcripts that contain an internal UGA codon, which greatly expands the potential mammalian targets that could be undergoing stop codon recoding or some form of PTR. 


\section{Materials and Methods}

\subsection{CUT tool algorithms and database design}

The CUT tool was implemented using a standard "Model View Controller" (MVC) software design pattern as a Java 2 Enterprise Edition (J2EE) package deployed on a JBoss application server. The "Model" is comprised of a MySQL relational database and Enterprise Java Bean (EJB), the "View" is provided by Java Server Pages (JSPs), and the "Controller" is formed by Java Servlets. Offline Java code was written to populate the database by parsing GTF annotation files (obtained from SGD and the UCSC table browser) and using this data to assemble coding sequences for each protein coding gene or transcript from the relevant genome sequences. The coding sequences then undergo a brief quality "control" check (e.g., ensure complete reading frame and accepted start codons). Accepted coding sequences were then analyzed for specific codon and codon combination usage. Codon sequence bias (for every contiguous combination of length 1 to 5 ) in each transcript is calculated as a Z-score (number of standard deviations above/below the population mean) by comparison to the full population of transcripts within a particular annotation set. The procedure for calculating the expected codon frequency for each gene/transcript was based on the observed global frequency of each mono-, di-, tri-, quadra- and quint-codon sequence in the set of all gene/transcripts in a given species. In addition, we treated all alternative isoforms of a given gene as a separate transcript, which resulted in the exon sequences found in each isoform being counted. We reasoned that multiple isoforms could be present in the cell at the same time, to support our use of codon sequences specific to each isoform as separate variables that compose the expected frequency value. Z-scores are a simple measure of whether a gene/transcript is using a codon sequence more or less then the global frequency. Specifically Z-scores detail how many standard deviations above or below the global frequency the specific codon sequence is used in the gene/transcript, which can be misleading when dealing with expected frequencies that approach zero. For example the Z-score measures for quintuplet codon combinations should be used with caution as the expected frequency of all quintuplet codons approaches zero and a single use in a gene/transcript results in a Z-score $>100$. We note that the number of times each quintuplet codon is used in a gene is detailed in the CUT database and these can be more informative measures then Z-scores. The online tool does not support right clicking due its use of dynamically generated gene/transcript pages. 


\subsection{Gene specific codon and dicodon analysis}

Species-containing genes or transcript data stored in the CUT database can be accessed using the search tab found at http://pare.sunycnse.com/cut/controller?action=search. The organism, genome, annotation and gene of interest must be specified in the pull down menu, with the organism designation leading to single genome and annotation choices. The gene/tx pull-down can be searched with partial or full gene names or transcript (NM_numbers) identifiers using the search gene button. Results on each search are displayed below the search gene button and their CUT data can be accessed by clicking on the gene/tx hyperlink, which will bring users to a page that displays the gene sequence and provides access to monocodon to quintuplecodon data and graphs for each gene. In addition there is a download spreadsheet tab for each gene that allows for the export of all monocodon and dicodon data that is linked to the gene-specific codon sequence, Z-score, expected frequency and actual frequency. The resulting download is produced in tab-delimited form. Resulting data can be imported into a graphing program (i.e., Excel) and data can be organized to identify the most and least used moncodons and dicodons in the gene sequence. On each gene/transcript webpage the hyperlinks detailing the monocodon to quintupletcodons used in each gene can be clicked to bring the user to bar graphs detailing the sequence, number of times used and Z-score.

\subsection{Gene codon painting and immunoblot analysis}

Codon painting can be performed using the codon search input box found on each gene/transcript page tied to the database. Once the specific codon pattern is searched (using the input field to specify sequence, search button to search and then highlight selected tab to paint), resulting output is highlighted to paint sequence occurrences in each target sequence. Protein expression analysis can be performed in the organism of choice. In the past in mouse and yeast models reported here we have analyzed specific proteins in the presence or absence of specific anticodon wobble base modifications [8-11, 13, 30]. For example, in yeast specific protein levels in wild-type (By4741) and trm9 4 cells were determined as previously described 
using corresponding C-terminal TAP tagged strains [31] and immunoblots. Briefly, each TAP-tag strain was made trm9 4 using a PCR amplified cassette derived from YML014W, with selection occurring on G418. Protein extracts from each strain were then analyzed by immunoblots using an anti-TAP antibody, with equal loading probed using an anti- $\beta$-tubulin antibody, as previously described [13].

\subsection{CUT downloads, gene parsing and dicodon statistical analysis}

Using the Genomes tab, users can access a page that allows for the download of all gene-specific moncodon and dicodon data for yeast, mouse and rat genes/transcripts. Data on genome wide monocodon and dicodon frequencies for all species-containing genes/transcripts can be downloaded from the CUT database (http://pare.sunycnse.com/cut/controller?action=listAnnot) by clicking on the Download Stats button. The Heatmap Files icon that is shown on each gene/transcript page can be used to download all monocodon and dicodon Z-scores for all genes/transcripts in a specific organism. The resulting download is a compressed folder that when extracted will provide a tab-delimited file detailing Z-scores for all monocodons and dicodons used in each gene. Outside of CUT these lists can be used to compile sets of genes (i.e., visual basic programmed search of gene list in Excel) and, by using random sampling approaches, determine if summed Zscores for sets of genes are higher than average values. For example, visual basic based scripts can be programmed in Excel to randomly order all yeast genes, pick 80 random genes to obtain and then sum dicodon Z-score values, with this process performed for $\mathrm{N}=300$ occurrences. The average value and standard deviation for the summed Z-score can then be compared to an actual value to determine a measure of significance (Z-score), similar to our previously described studies [13, 32].

\subsection{Multi-codon searches to identify species-containing genes with specific in frame sequences}

Multi-codon searches to identify species-containing genes with specific sequence can also be performed in the CUT database using the search tab (http://pare.sunycnse.com/cut/controller?action=search). The organism, genome and annotation information must be specified in the pull down menu, with the organism designation 
only providing a single genome and annotation choice. The "containing codon sequence" input can be specified with a codon string (i.e., UGANNN, with $\mathrm{N}$ being either $\mathrm{U}, \mathrm{G}, \mathrm{A}$ or $\mathrm{C}$ ), followed by a click on the search gene button. Results of each search are displayed below the search gene button and their CUT data can be accessed by clicking on the gene/tx hyperlink, which will bring users to a page that displays gene sequence and other information, as described above, for each gene.

\section{Results and Discussion}

The CUT algorithm was developed to methodically count the codon content in gene or transcript sequences beginning with the start codon. To facilitate bulk gene analysis, data was imported (Fig. 1) as either gene annotations (SGD, 6664 yeast genes) or as RefSeq annotated transcripts (30,392 mouse and 16,711 rat transcripts). A key difference between the yeast and mammalian data imports is that genes are represented as single entities, while multiple transcripts are present for specific genes, respectively, with the latter including splice variants. Each entry was methodically analyzed to catalog the number of each possible

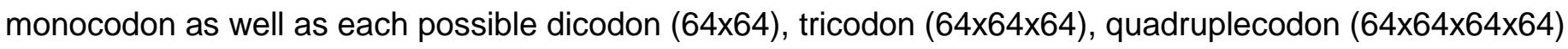
and quintuplecodon $(64 \times 64 \times 64 \times 64 \times 64)$ combinations (http://pare.sunycnse.com/cut/). For each gene/transcript, there are 64 to over 1 billion possible features to catalog and store, but no gene had codon sequences that represent the entire combination space. The CUT database was designed using a table based format (Fig. 2) and allows for individual gene queries and the retrieval of all of the codon combination data noted earlier, and describes whether a codon or codon combination is over-represented in a gene sequence using a Z-score metric. Search functions that are associated with CUT include queries based on Z-score cutoffs, codon combinations, and gene identity. Bulk download of codon usage frequencies and Z-scores for all monocodon and dicodon combinations is enabled for individual genes as well as for all genes or transcripts specific to each species.

We have previously reported that the yeast translation elongation factor 3 (YEF3) transcript is translationally regulated by Trm9-catalyzed uridine modifications on tRNA and that YEF3 overuses GAA (91 total count, 3.4 Z-score) and AGA (41 total count, 3.6 Z-score) codons [13, 33]. In trm9 4 cells, we have shown 
that Yef3 protein levels are dramatically reduced, with little difference observed in transcript levels [10, 13]. YEF3 was used to query the database (Fig. 3A) to demonstrate results that report monocodon usage patterns in bar graph format (Fig. 3B). In addition, YEF3 was searched to detail dicodon usage pattern numbers (Fig. 3C), such as GAA-GAA, which is represented 8 times and has a Z-score of 3.2. Z-scores that detail pattern usage can be exported for all represented dicodons in a gene. For example, data specific to YEF3 have been exported, sorted from low to high, and plotted in Fig. 3D. Dicodons overused in YEF3 are found on the far right side of Fig. 3D and have Z-scores $>8$. It is interesting to note that 22 of the 25 most overused dicodons in YEF3 contain either GAA or AGA, with both codons decoded by tRNAs modified by Trm9. Notably, the dicodon combinations AGA-AGA, GAA-AGA, AGA-GAA and GAA-GAA were in the top 25 most overrepresented list for YEF3, having Z-scores of 4.8, 4.1, 3.3 and 3.2, respectively. We propose that dicodon patterns are important because they report on the specific tRNAs that will need to be sequentially accessed for translation. In the case of AGA-AGA, GAA-AGA, AGA-GAA, GAA-GAA, they are all decoded by tRNAs modified by Trm9, such that these dicodons should be difficult to translate if there is a deficiency in Trm9dependent uridine modifications.

We have also used CUT to explore codon usage patterns in yeast genes (Fig. 4) other then YEF3. Our goal was to determine if we could highlight significant dicodon and quintuplecodon patterns that correlate with decreased protein levels in trm9 $\Delta$ cells, relative to wild-type cells. Specifically, we have used the codon painting function of CUT to highlight the AGA codon in the gene ENT2 (Fig. 4A), with the finding that this gene contains two AGA-AGA doublets $(Z$-score $=2.0)$. The Ent2 protein is required for actin patch assembly and endocytosis. Based on over-use of AGA-AGA dicodons, we predicted that Ent3 protein levels would decrease in trm9 4 cells, a prediction that was confirmed by immunoblot analysis (Fig. 4B). As a negative control we have included immunoblot data on Met6 protein levels from wild-type and trm9 4 cells (Supplemental Figure S1). The Met6 gene is over-represented with AGA codons (31 total, Z-score $=2.5$ ) but contains 0 AGA-AGA dicodons. Met6 protein levels are not affected by a Trm9 deficiency, further supporting the idea that complex codon patterns are an important determinant of MoTTs.

The GAA codon has also been shown to have its translation linked to Trm9. We searched the CUT database to identify genes that use five GAA codons in a row and identified NAB3. In Fig. 4C, we painted 
NAB3 to identify GAA codons, with the finding that there are nine instances of five GAA codons in a row (Zscore $=28$ ). These nine instances were found in runs of 7 and 10 GAA codons. Nab3 is an RNA-binding protein that is part of the Nrd1 complex involved in some 3'-end processing. The GAA codon runs found in Nab3 are extremely rare and, based on previously published reporter data, the Nab3 protein should be decreased in trm9 4 cells compared to wild-type, which we confirmed by immunoblot analysis (Fig. 4D).

Translation of the AAG codon has been linked to Trm9 [9]. We have previously used an AAG-AAGAAG-AAG reporter system to observe a modest but statistically insignificant decrease in reporter activity in trm9 $\Delta$ cells, relative to wild-type [9]. The reporter system is an artificial construct and we wanted to determine if any endogenous yeast genes had AAG codon combinations greater than four and, if so, was the corresponding protein expression linked to Trm9. We used the CUT database to find the HMO1 gene, which was significantly over-represented with AAG-AAG-AAG-AAG-AAG. This quintuplecodon occurred four times, with a Z-score of 55 (Fig. 4E). In fact, all four AAG-AAG-AAG-AAG-AAG quintuple codons are found in runs of 8 AAG codons. We predicted that, due to the presence of 8 AAG codons in a row, the levels of the Hmo1 protein should be strongly dependent on Trm9, with was again supported by immunoblot data (Fig. 4F). The HMO1 gene is an interesting case because it contains 23 AAG codons in total, which is an average amount. The significance of the AAG codon in NAB3 becomes apparent when dicodons are analyzed, with the AAGAAG dicodon occurring 10 times and has a Z-score of 8.0. NAB3 represents a good example of how complex codon patterns can be more informative for modeling translation than simple monocodon based descriptors.

In some cases, regulation of gene expression requires the coordination of many genes and, based on monocodon patterns, we have previously reported that groups of genes have the potential to be regulated by changes in tRNA wobble base modifications. The CUT database can be used to download all dicodon data for all genes in a species and determine if groups of genes over-use some dicodon combinations. In response to nutrient starvation, the Gcn2-elF2 $\alpha-G c n 4$ pathway is activated by translational regulation [34-39], with subsequent Gcn4-based transcriptional regulation of many genes. Computational studies have identified Gcn4-binding sites in 80 transcripts [40]. We identified the 80 Gcn4-regulated genes in the CUT database download detailing dicodon usage, and then summed the Z-scores in each dicodon category, to get a group measure of dicodon over-usage (Fig. 5). The five dicodons with the highest summed Z-scores (>65) for the 
group of 80 Gcn4-regulated transcripts were GGU-GCC (Gly-Gly), GCA-GGU (Ala-Gly), GCU-GUG (Ala-Val), GGU-GUU (Gly-Val) and AGA-GAA (Arg-Glu). We were intrigued that the Trm9-regulated codons AGA and GAA were found in the list of top-scoring dicodons for the $80 \mathrm{Gcn} 4$ transcripts, so we used a random sampling of 80 transcripts (300 times from all yeast genes) to determine significance (Fig. 5, inset). We note that significance of a group score was performed outside of CUT and this functionality is planned for later versions of the tool. The average summed AGA-GAA dicodon Z-score for 80 randomly-sampled genes was 30 with a standard deviation of 7.2, making a highly significant $\left(P<10^{-5}\right)$ Z-score of 68 for Gcn4-regulated transcripts. While we can directly link the AGA-GAA dicodon to regulation by Trm9, the other top scoring Gly-, Ala- and Val-based codons are dependent on other tRNAs. It is interesting that the Gcn2-elF2 $\alpha$-Gcn4 pathway, which begins with regulation of translation initiation, can now be linked to regulation of translation elongation through the AGA-GAA dicodon.

Next we determined if the CUT tool and database could used to identify interesting codon trends in mammalian transcripts. Specifically, we have applied the same algorithms and developed the same database query forms used to study yeast genes and analyzed all transcripts available in the RefSeq datasets for mice and rat genes (Fig. 6A). We highlight mouse Brca1 and Gpx1 genes, which encode key stress response proteins whose activities play important roles in DNA repair and ROS-detoxification, respectively [41, 42]. We downloaded dicodon Z-score data for all Brca1 (Fig. 6B) and Gpx1 entries (Fig. 6A) and then visualized the gene specific data in a scatter plot. Note that the maximum value for the Y-axis is 25 for Brca1 and 65 for Gpx1. As can be observed in both Brca1 and Gpx1 plots (Fig. 6B-C), most of the data stays on a similar horizontal plane near the Y-axis (Z-score) values of 0 to 10 (Brca1) and 0 to 5 (Gpx1). There are a number of dicodon outliers for Brca1 that have Z-scores ( 22), including AGU-AAA and UUA-CCG (Fig. 6B). A similar analysis of Gpx1 was performed (Fig. 6C), with the most dramatic outlier found at a Z-score of 55 for UGAGGC, which was twice the score of any dicodon found in Brca1. The high Z-score for the UGA-GGC dicodon in Gpx1 was expected as the corresponding protein contains selenocysteine, which is incorporated by stop codon recoding and utilizes a non standard internal UGA. Gpx1 and the UGA-GGC dicodon are examples of extreme codon usage, as there are only 24 reported selenoproteins in mice [43], and UGA is not usually followed by another codon in most transcripts, because UGA usually signals for the end of translation. 
In addition to using CUT to identify significant codon patterns in individual or groups of genes, the program is also designed to be used as an exploration tool. We decided to use CUT to identify all mouse and rat transcripts that contain an internal UGA codon (Fig. 7), as this should identify transcripts that have the potential to undergo stop codon recoding (i.e., selenoproteins) or to participate in some form of translational read-through to generate an extended protein with a different function (e.g., VegF-Ax) [25] or targeting to a specific organelle (Mdh1) [27]. We searched both the mouse and rat data loaded in CUT using the sequence UGA-NNN, where NNN represents any of the 64 codons. Our search identified 45 mouse (Table I) and 120 rat (Table II) transcripts with internal UGA codons, which was more than the 25 - 30 expected hits for each organism. We had expected 23-24 selenoproteins and a small number of other reported transcripts that undergo translational read-through in mice and rats. The number of internal UGA transcripts noted for each of our identified targets ranged from 1 to 20 for mouse transcripts and 1 to 36 for rat transcripts. While the transcripts that contain more than 1 UGA codon could be RefSeq annotation errors, we note that transcripts for selenoproteins can include 1 to 10 internal UGA codons [12, 43], with Sepp1 corresponding to the latter in both our mouse and rat datasets (Tables I and II).

Specific to the mouse UGA-NNN search using the CUT tool, we identified all 24 known mouse selenoproteins as well as an additional 21 transcripts with internal UGA codons. We speculate that the 21 mouse transcripts have their UGA codon translated, with this speculation supported for 3 below detailed proteins. Specific to the rat UGA-NNN search using the CUT tool, we identified 23 of 24 known rodent selenoproteins, with the exception of RGD1560938/Seli/Ept1, which does not have an internal UGA codon. Remarkably, we identified an additional 97 transcripts with internal UGA codons. All identified mouse and rat transcripts were compared to the NCBI database using a nucleotide blast (http://blast.ncbi.nlm.nih.gov/Blast.cgi) to ensure correct gene identification, with criteria of $>99 \%$ identical gene BLAST being employed. The biochemical functions of selenoproteins include reduction of ROS, selenium transport and selenocysteine synthesis. Additionally, some selenoproteins play major roles in cancer prevention, thyroid metabolism, male fertility, and immune, muscle, and central nervous system functions [4]. The roles of some selenoproteins such as selenoprotein O, T and H (identified by CUT as 2700094K13Rik), are still unknown. 
Mouse- and rat-specific transcripts that possess internal UGA codons but do not contain selenocysteine were also most likely identified in our UGA-NNN search. For example, VegFA was identified in our mouse and rat searches. The human version of VegF, known as VegF-Ax, was reported by Fox and co-workers to use translational read-through of the UGA codon to form a longer protein with anti-angiogenic activity [25]. In addition, studies have provided evidence for UGA read-through targets identified in our search, and they included Ago1 and Mtch2 [25], as well as $L d h B$ and Mdh1 (mouse and rat) [27]. In some cases, specific cisacting sequence elements are believed to facilitate UGA read-through, with the resulting C-terminally extended proteins taking on physiologically distinct roles compared to those produced using the upstream (or canonical) UGA. For VegF-Ax (a VegF-A isoform with 22 additional C-terminal amino acids), the ribonuclear protein A2/B1 was identified as a trans-acting factor critical for VegF-Ax production. In parallel, for selenocysteinedecoding transcripts, recognition of the SECIS element by the RNA binding protein Sbp2 is critical for UGA translation as selenocysteine [44]. 3'-UTR and other bioinformatics parameters that can be used to further parse the identified UGA-containing transcripts are discussed below.

LdhB and Mdh1 can both be considered stress-response enzymes critical for intracellular redox homeostasis and they have CU bases immediately following the UGA stop codon. It has been shown that changing the $\mathrm{CU}$ dinucleoteide results in decreased read-through efficiency, as does changing the stop codon [27]. Lactate dehydrogenase $B(L d h B)$ is one subunit of the lactate dehydrogenase enzyme, which converts lactate to pyruvate and vice versa via the interconversion of $\mathrm{NADH}$ and $\mathrm{NAD}^{+}$. The C-terminally extended LdhB, termed LdhBx, has 6 extra amino acids and contains a peroxisomal targeting signal type 1 (PTS1) sequence in the $3^{\prime}$ extension. LdhBx has also been shown to co-import LdhA, another lactate dehydrogenase subunit, into peroxisomes [45]. Similarly, two protein isoforms of malate dehydrogenase 1 (Mdh1) exist, one of which is created via translational read-through to create a 20 amino acid C-terminally extended protein. Mdh1 catalyzes the reversible oxidation of malate to oxaloacetate using $\mathrm{NADH} / \mathrm{NAD}^{+}$, with the extended protein also containing a PTS1 [2]. It is therefore likely that peroxisomal localization of both LdhB and Mdh1 is dependent on stop codon read-through.

Genome-wide analysis and published reports have identified two additional targets of translational read-through, Ago1 and Mtch2, which contain 37 and 10 amino acids, respectively. Argonaute 1 (Ago1) is 
involved in RNA interference and RNA silencing by binding to micro-RNAs or siRNAs and repressing translation of complementary mRNA [46]. Mitochondrial carrier 2 (Mtch2) is a key player in the mitochondrial death pathway via the recruitment of proapoptotic truncated BID (tBID) [47]. It is still unclear how translational read-through alters the function of these two proteins as they have only recently been identified as targets for PTR. Ago1 and Mtch2 do not share the same CU dicodon following UGA that is necessary for translational read-through of LdhB and Mdh1. Instead, both Ago1 and Mtch2 are followed by an "AG" dicodon.

Interestingly, the read-through efficiency of these two transcripts is highest among the five known PTR targets, at $13 \%$ for Mtch2 and 24\% for Ago1, compared to 11\% for VegF-Ax [25] and 1-2\% for LdhB and Mdh1 [45].

We identified 97 rat transcripts with internal UGA codons that are not currently classified as selenoproteins. Similar to the mouse results we speculate that these UGA codons are translated. Considering the links between toxicant-induced tRNA modifications and the translational decoding of stress response transcripts,[48] several of these gene transcripts stand out. For example, Bag4 belongs to a family of Bagrelated chaperone proteins that have been shown to regulate cell death and growth decisions by interacting with both anti-apoptotic factors, as well as growth stimulatory kinases such as PI3K [49-52]. Other internal UGA-containing transcripts can be linked to cellular stress responses through their involvement in DNA damage-induced cell cycle checkpoint controls: the Cdc25b phosphatase targets cyclin dependent kinase 2 for dephosphorylation allowing entry into mitosis, and is itself targeted by Chk1 in response to DNA damage [53, 54]; the Taok1 kinase mediates chromosome-microtubule interactions during $\mathrm{M}$ phase of the cell cycle, guarding against chromosomal instability [55]. Last, Fam120a (also Ossa/C9orf10) is an RNA binding protein implicated in tumor cell resistance to oxidative stress by mechanisms thought to involve the activation of antiapoptotic signals [56]. It will be interesting to determine the extent of translational recoding during stress responses, as it could provide new activities to optimize the response.

A total of 32 rat olfactory receptors were identified as having at least one internal UGA codon, and several had greater than five. Rat olfactory proteins comprise seven-transmembrane receptors and sense smell via direct interaction with odorant molecules and they belong to the largest multi-gene family in rats [57]. Perhaps differential UGA recoding represents a further means of diversification for olfactory receptors in rats, contributing to their advanced ability to differentiate between a myriad of odorants. It is further remarkable to 
find such a high number of internal UGA-containing transcripts in rat compared to mouse. This finding raises the possibility that rat uses UGA recoding to diversify protein expression from an individual transcript to an extent greater than that observed in other rodents. It remains to be seen whether the internal UGA of these transcripts is decoded as selenocysteine, or serine, as is the case for $V e g F-A x[25,58]$. The first step has been taken in our methodical identification of transcripts with internal UGA codons as candidates for recoding; the next step will be to employ proteomic analysis of candidate transcripts in order to determine which amino acid is used. Additionally it will be interesting to investigate whether the UGA containing mouse and rat transcript genes are encoded by transcripts that display a further codon bias and whether their regulation is influenced by tRNA modifications, according to the MoTT hypothesis $[48,59]$.

\section{Conclusions}

We have demonstrated the utility of the CUT tool and database by applying and analyzing gene specific dicodon patterns in yeast, mice and rat genes and transcripts. Development of the CUT database has permitted the first genome-wide exhaustive analysis of internal UGA stop codons. Our results reveal a diversity of potential new transcripts that undergo translational recoding, which can provide a means to expand the genetic code and increase the functional diversity of gene products without requiring the addition of new genetic material. While our UGA-NNN search has identified many transcripts with the potential to be translationally decoded, bioinformatics analyses of functional classification, 3'-UTR sequence space, and conservation between species will need to be performed to add further proof. One approach that could be employed to validate that the UGA-NNN transcripts are translated could be mass spectrometry based proteomics to identify the corresponding peptide. In addition, experimental evidence that provides direct support for translational decoding in the form of new peptides that contain selenocysteine or that correspond to amino acid sequences downstream of standard stop codons will be needed to confirm that the UGA codon was translationally decoded. Nevertheless the CUT tool thus provides a powerful new approach to gain insight into the complexity of mono- and multi-codon usage across yeast and mammalian genomes which can be used to elucidate the biological function of codon usage patterns in the translational control of gene expression. The 
version 1 CUT tool and database provides simple and complex codon usage data for all yeast, mouse and rat genes/transcripts in their respective genomes. The future evolution of CUT will include the addition of specific modules that include human, bacterial and other model organism data sets. Complex codon usage patterns have the potential to be regulatory in many species, and the CUT tool and database approach has the potential to be applied to all sequenced and gene-defined genomes. Future capabilities that include advanced search functions based on gene function and codon count criteria, statistical analysis to identify significant codon patterns in groups of genes and genome-genome comparisons are also planned. 


\section{Acknowledgments}

We would like to thank past and present lab members and colleagues for helping us compile and think about the data. We would like to thank Fraulin Joseph for his work on immunoblots. Funding for the these studies was provided by NIH ES017010 (TJB), National Science Foundation CHE-1308839 (PCD), National Research Foundation of Singapore through the Singapore-MIT Alliance for Research and Technology Infectious Disease research program (PCD). 


\section{References}

[1] D. Globisch, D. Pearson, A. Hienzsch, T. Bruckl, M. Wagner, I. Thoma, P. Thumbs, V. Reiter, A.C. Kneuttinger, M. Muller, S.A. Sieber, T. Carell, Angew Chem Int Ed Engl, 50 (2011) 9739-9742.

[2] M.A. Machnicka, K. Milanowska, O. Osman Oglou, E. Purta, M. Kurkowska, A. Olchowik, W. Januszewski, S. Kalinowski, S. Dunin-Horkawicz, K.M. Rother, M. Helm, J.M. Bujnicki, H. Grosjean, Nucleic Acids Res, 41 (2013) D262-267.

[3] E.M. Novoa, M. Pavon-Eternod, T. Pan, L. Ribas de Pouplana, Cell, 149 (2012) 202-213.

[4] P.F. Agris, F.A. Vendeix, W.D. Graham, J Mol Biol, 366 (2007) 1-13.

[5] S. Tikole, R. Sankararamakrishnan, Journal of biomolecular structure \& dynamics, 24 (2006) 33-42.

[6] J.S. Weinger, K.M. Parnell, S. Dorner, R. Green, S.A. Strobel, Nature structural \& molecular biology, 11 (2004) 1101-1106.

[7] D.L. Oxender, G. Zurawski, C. Yanofsky, Proc Natl Acad Sci U S A, 76 (1979) 5524-5528.

[8] C.T. Chan, Y.L. Pang, W. Deng, I.R. Babu, M. Dyavaiah, T.J. Begley, P.C. Dedon, Nat Commun, 3 (2012) 937.

[9] A. Patil, M. Dyavaiah, F. Joseph, J.P. Rooney, C.T. Chan, P.C. Dedon, T.J. Begley, Cell Cycle, 11 (2012) 3656-3665.

[10] W. Deng, I.R. Babu, D. Su, S. Yin, T.J. Begley, P.C. Dedon, PLoS Genet, 11 (2015) e1005706.

[11] L. Endres, U. Begley, R. Clark, C. Gu, A. Dziergowska, A. Malkiewicz, J.A. Melendez, P.C. Dedon, T.J. Begley, PLoS One, 10 (2015) e0131335.

[12] V.N. Gladyshev, D.L. Hatfield, J Biomed Sci, 6 (1999) 151-160. 
[13] U. Begley, M. Dyavaiah, A. Patil, J.P. Rooney, D. Direnzo, C.M. Young, D.S. Conklin, R.S. Zitomer, T.J. Begley, Mol Cell, 28 (2007) 860-870.

[14] T.Y.C. Chan, W. Deng, F. Li, M.S. DeMott, I.R. Babu, T.J. Begley, P.C. Dedon, Chem Res Toxicol, 28 (2015) $978-988$.

[15] C.T. Chan, M. Dyavaiah, M.S. DeMott, K. Taghizadeh, P.C. Dedon, T.J. Begley, PLoS Genet, 6 (2010) e1001247.

[16] C.T.Y. Chan, Y.L.J. Pang, W. Deng, I.R. Babu, M. Dyavaiah, T.J. Begley, P.C. Dedon, Nat Commun, 3 (2012) 937.

[17] M.E. Moustafa, B.A. Carlson, M.A. El-Saadani, G.V. Kryukov, Q.A. Sun, J.W. Harney, K.E. Hill, G.F. Combs, L. Feigenbaum, D.B. Mansur, R.F. Burk, M.J. Berry, A.M. Diamond, B.J. Lee, V.N. Gladyshev, D.L. Hatfield, Mol Cell Biol, 21 (2001) 3840-3852.

[18] A. Bock, K. Forchhammer, J. Heider, W. Leinfelder, G. Sawers, B. Veprek, F. Zinoni, Mol Microbiol, 5 (1991) 515-520.

[19] B.J. Lee, P.J. Worland, J.N. Davis, T.C. Stadtman, D.L. Hatfield, J Biol Chem, 264 (1989) 9724-9727.

[20] V.N. Gladyshev, D.L. Hatfield, Curr Protoc Protein Sci, Chapter 3 (2001) Unit 38.

[21] S.V. Novoselov, D.F. Calvisi, V.M. Labunskyy, V.M. Factor, B.A. Carlson, D.E. Fomenko, M.E. Moustafa, D.L. Hatfield, V.N. Gladyshev, Oncogene, 24 (2005) 8003-8011.

[22] L. Songe-Moller, E. van den Born, V. Leihne, C.B. Vagbo, T. Kristoffersen, H.E. Krokan, F. Kirpekar, P.O. Falnes, A. Klungland, Mol Cell Biol, 30 (2010) 1814-1827.

[23] M.J. Berry, L. Banu, J.W. Harney, P.R. Larsen, EMBO J, 12 (1993) 3315-3322.

[24] K.V. Korotkov, S.V. Novoselov, D.L. Hatfield, V.N. Gladyshev, Mol Cell Biol, 22 (2002) 1402-1411. 
[25] S.M. Eswarappa, A.A. Potdar, W.J. Koch, Y. Fan, K. Vasu, D. Lindner, B. Willard, L.M. Graham, P.E. DiCorleto, P.L. Fox, Cell, 157 (2014) 1605-1618.

[26] A. Hoeben, B. Landuyt, M.S. Highley, H. Wildiers, A.T. Van Oosterom, E.A. De Bruijn, Pharmacological reviews, 56 (2004) 549-580.

[27] A.C. Stiebler, J. Freitag, K.O. Schink, T. Stehlik, B.A. Tillmann, J. Ast, M. Bolker, PLoS Genet, 10 (2014) e1004685.

[28] T. Ikemura, Mol Biol Evol, 2 (1985) 13-34.

[29] E.B. Keller, J.M. Calvo, Proc Natl Acad Sci U S A, 76 (1979) 6186-6190.

[30] A. Patil, C.T. Chan, M. Dyavaiah, J.P. Rooney, P.C. Dedon, T.J. Begley, RNA Biology, 9 (2012) 990 1001.

[31] S. Ghaemmaghami, W.K. Huh, K. Bower, R.W. Howson, A. Belle, N. Dephoure, E.K. O'Shea, J.S. Weissman, Nature, 425 (2003) 737-741.

[32] T.J. Begley, A.S. Rosenbach, T. Ideker, L.D. Samson, Mol Cell, 16 (2004) 117-125.

[33] S. Tumu, A. Patil, W.L. Towns, M. Dyavaiah, T.J. Begley, Database, (2012) bas002.

[34] A.G. Hinnebusch, K. Natarajan, Eukaryot Cell, 1 (2002) 22-32.

[35] K. Natarajan, M.R. Meyer, B.M. Jackson, D. Slade, C. Roberts, A.G. Hinnebusch, M.J. Marton, Mol Cell Biol, 21 (2001) 4347-4368.

[36] A. Goossens, T.E. Dever, A. Pascual-Ahuir, R. Serrano, J Biol Chem, 276 (2001) 30753-30760.

[37] R. Yang, S.A. Wek, R.C. Wek, Mol Cell Biol, 20 (2000) 2706-2717.

[38] A.G. Hinnebusch, Mol Microbiol, 10 (1993) 215-223.

[39] T.E. Dever, L. Feng, R.C. Wek, A.M. Cigan, T.F. Donahue, A.G. Hinnebusch, Cell, 68 (1992) 585-596. 
[40] O. Schuldiner, C. Yanover, N. Benvenisty, Current genetics, 33 (1998) 16-20.

[41] P.L. Welcsh, K.N. Owens, I. King, Trends Genet., 16 (2000) 69-74.

[42] J.B. de Haan, C. Bladier, P. Griffiths, M. Kelner, R.D. O'Shea, N.S. Cheung, R.T. Bronson, M.J. Silvestro, S. Wild, S.S. Zheng, P.M. Beart, P.J. Hertzog, I. Kola, J Biol Chem, 273 (1998) 22528-22536.

[43] G.V. Kryukov, S. Castellano, S.V. Novoselov, A.V. Lobanov, O. Zehtab, R. Guigo, V.N. Gladyshev, Science, 300 (2003) 1439-1443.

[44] D.M. Driscoll, P.R. Copeland, Annu Rev Nutr, 23 (2003) 17-40.

[45] F. Schueren, T. Lingner, R. George, J. Hofhuis, C. Dickel, J. Gartner, S. Thoms, eLife, 3 (2014) e03640.

[46] D.H. Kim, L.M. Villeneuve, K.V. Morris, J.J. Rossi, Nature structural \& molecular biology, 13 (2006) 793797.

[47] C. Katz, Y. Zaltsman-Amir, Y. Mostizky, N. Kollet, A. Gross, A. Friedler, J Biol Chem, 287 (2012) $15016-$ 15023.

[48] L. Endres, P.C. Dedon, T.J. Begley, RNA Biol, 12 (2015) 603-614.

[49] M. Boiani, C. Daniel, X. Liu, M.D. Hogarty, L.J. Marnett, J Biol Chem, 288 (2013) 6980-6990.

[50] P. Rahman, R.D. Huysmans, F. Wiradjaja, R. Gurung, L.M. Ooms, D.A. Sheffield, J.M. Dyson, M.J. Layton, A. Sriratana, H. Takada, T. Tiganis, C.A. Mitchell, J Biol Chem, 286 (2011) 29758-29770.

[51] S. Takayama, Z. Xie, J.C. Reed, J Biol Chem, 274 (1999) 781-786.

[52] H. Eichholtz-Wirth, E. Fritz, L. Wolz, Cancer Lett, 194 (2003) 81-89.

[53] Y. Sanchez, C. Wong, R.S. Thoma, R. Richman, Z. Wu, H. Piwnica-Worms, S.J. Elledge, Science, 277 (1997) 1497-1501.

[54] G. Draetta, J. Eckstein, Biochim Biophys Acta, 1332 (1997) M53-63. 
[55] R.L. Shrestha, N. Tamura, A. Fries, N. Levin, J. Clark, V.M. Draviam, Open biology, 4 (2014) 130108.

[56] M. Tanaka, K. Sasaki, R. Kamata, Y. Hoshino, K. Yanagihara, R. Sakai, Mol Cell Biol, 29 (2009) 402-413.

[57] L.B. Buck, Cell, 116 (2004) S117-119, 111 p following S119.

[58] S.M. Eswarappa, P.L. Fox, Cancer Res, 75 (2015) 2765-2769.

[59] P.C. Dedon, T.J. Begley, Chem Res Toxicol, 17 (2014) 7. 
Table I. CUT-identified Mouse Transcripts with Internal UGA Codons

\begin{tabular}{|c|c|c|c|c|c|}
\hline $\begin{array}{c}\text { Mouse Gene } \\
\text { Name }\end{array}$ & $\begin{array}{l}\text { \# of internal } \\
\text { UGA codons }\end{array}$ & Transcript ID & $\begin{array}{c}\text { Mouse Gene } \\
\text { Name }\end{array}$ & $\begin{array}{c}\text { \# of internal UGA } \\
\text { codons }\end{array}$ & Transcript ID \\
\hline 4933416I08Rik & 9 & NM_027700 & Msrb1 & 1 & NM_013759 \\
\hline 2700094K13Rik & 1 & NM_001033166 & Mtch2 & 1 & NM_001317241 \\
\hline Ago1 & 1 & NM_001317173 & Oas1b & 1 & NM_001083925 \\
\hline Art2a-ps & 1 & NM_007490 & Olfr421-ps1 & 1 & NM_146720 \\
\hline BC089491 & 1 & NM_175033 & Olfr915 & 1 & NM_146785 \\
\hline Clec7a & 1 & NM_020008 & Pira6 & 6 & NM_011093 \\
\hline Dio1 & 1 & NM_007860 & Ptprv & 1 & NM_007955 \\
\hline Dio2 & 2 & NM_010050 & Selk & 1 & NM_019979 \\
\hline Dio3 & 1 & NM_172119 & Selm & 1 & NM_053267 \\
\hline Ept1 & 1 & NM_027652 & Selo & 1 & NM_027905 \\
\hline Gm10058 & 1 & NM_001109969 & Selt & 1 & NM_001040396 \\
\hline Gm10096 & 1 & NM_001102678 & Sep15 & 1 & NM_053102 \\
\hline Gm10147 & 1 & NM_001099919 & Sephs2 & 1 & NM_009266 \\
\hline Gm10230 & 1 & NM_001099347 & Sepn1 & 1 & NM_029100 \\
\hline Gm10486 & 1 & NM_001109970 & Sepp1 & 10 & NM_009155 \\
\hline Gm14819 & 1 & NM_001110250 & Sepw1 & 1 & NM_009156 \\
\hline Gpx1 & 1 & NM_008160 & Smcp & 3 & NM_008574 \\
\hline Gpx2 & 1 & NM_030677 & Txnrd1 & 1 & NM_015762 \\
\hline Gpx3 & 1 & NM_008161 & Txnrd2 & 1 & NM_013711 \\
\hline Gpx4 & 1 & NM_008162 & Txnrd3 & 1 & NM_153162 \\
\hline Ldhb & 1 & NM_001316322 & Vegfa & 1 & NM_001317041 \\
\hline Mdh1 & 1 & NM_001316675 & Vimp & 1 & NM_024439 \\
\hline Mia3 & 20 & NM_177389 & & & \\
\hline
\end{tabular}


Table II. CUT-identified Rat Transcripts with Internal UGA Codons

\begin{tabular}{|c|c|c|c|c|c|}
\hline Rat Gene Name & $\begin{array}{l}\text { \# of internal } \\
\text { UGA codons }\end{array}$ & Transcript ID & Rat Gene Name & $\begin{array}{l}\text { \# of internal } \\
\text { UGA codons }\end{array}$ & Transcript ID \\
\hline Abcb11 & 6 & NM_031760 & $\mathrm{Hdx}$ & 26 & NM_001134568 \\
\hline Aass & 12 & NM_001100963 & Impg1 & 5 & NM_023958 \\
\hline Abca1 & 8 & NM_178095 & Itpr3 & 12 & NM_013138 \\
\hline Abi2 & 12 & NM_173143 & $\mathrm{Kb} 23$ & 1 & NM_001008813 \\
\hline Acat2 & 1 & NM_001006995 & Kbtbd7 & 1 & NM_001302944 \\
\hline Acp1 & 4 & NM_001313735 & Kif1c & 14 & NM_145877 \\
\hline Aldh1a7 & 6 & NM_017272 & Kng2 & 4 & NM_001102418 \\
\hline Atn1 & 10 & NM_017228 & Krt75 & 1 & NM_001008828 \\
\hline Bag4 & 9 & NM_001025130 & Krt77 & 1 & NM_001008807 \\
\hline Batf3 & 1 & NM_021865 & Ldhb & 1 & NM_001316333 \\
\hline Cacna1a & 9 & NM_012918 & LOC100911576 & 4 & NM_001271241 \\
\hline Ccd25b & 10 & NM_133572 & LOC498592 & 6 & NM_001166307 \\
\hline Ces1e & 9 & NM_031565 & LOC500684 & 12 & NM_001047959 \\
\hline Ces2a & 3 & NM_144743 & Lrp1b & 2 & NM_001107843 \\
\hline Cntnap5a & 5 & NM_001047865 & Ly6c & 3 & NM_020103 \\
\hline Cstf3 & 7 & NM_001077672 & Mdh1 & 1 & NM_001316877 \\
\hline Сур3а9 & 1 & NM_147206 & Mfsd14a & 17 & NM_001106467 \\
\hline Dio1 & 1 & NM_021653 & Msrb1 & 1 & NM_001044285 \\
\hline Dio2 & 2 & NM_031720 & Mybpc1 & 14 & NM_001100758 \\
\hline Dio3 & 1 & NM_017210 & Ndrg3 & 3 & NM_001013923 \\
\hline Doc2b & 8 & NM_031142 & Nkr-plc & 1 & NM_001040189 \\
\hline Dync2h1 & 2 & NM_023024 & Olr1000 & 1 & NM_001000077 \\
\hline Eml1 & 8 & NM_001025741 & Olr1052 & 4 & NM_001001363 \\
\hline Fam120a & 2 & NM_001191816 & Olr1064 & 6 & NM_001001076 \\
\hline Fdsp & 1 & NM_031840 & Olr1065 & 5 & NM_001000498 \\
\hline Gnb5 & 1 & NM_031770 & Olr1065 & 5 & NM_001000498 \\
\hline Gpx1 & 1 & NM_030826 & Olr1226 & 8 & NM_001000442 \\
\hline Gpx2 & 1 & NM_183403 & Olr1227 & 6 & NM_001000443 \\
\hline Gpx3 & 1 & NM_022525 & Olr1228 & 5 & NM_001000964 \\
\hline Gpx4 & 1 & NM_001039849 & Olr1229 & 10 & NM_001000444 \\
\hline Grcc10 & 3 & NM_001198725 & Olr1237 & 8 & NM_001000811 \\
\hline
\end{tabular}


Table II (continued). CUT-identified Rat Transcripts with Internal UGA Codons (continued)

\begin{tabular}{|c|c|c|c|c|c|}
\hline Rat\$̧Gene\$Name & $\begin{array}{l}\text { \#\$f\$nternal\$ } \\
\text { UGAșodons }\end{array}$ & Transcript\$D & Rat\$̧Gene\$Name & $\begin{array}{l}\text { \#\$̣f\$়'nternal\$ } \\
\text { UGAșodons }\end{array}$ & Transcript\$D \\
\hline Olr1238 & 7 & NM_001001013 & RGD1307621 & 1 & NM_001108025 \\
\hline Olr1239 & 8 & NM_001000811 & RGD1563348 & 1 & NM_001114939 \\
\hline Olr1240 & 6 & NM_001000448 & Sell & 1 & NM_001134754 \\
\hline Olr1241 & 6 & NM_001000449 & Selk & 1 & NM_207589 \\
\hline Olr1247 & 7 & NM_001000807 & SelM & 1 & NM_001115013 \\
\hline Olr1254 & 7 & NM_001001085 & SelO & 1 & NM_001085485 \\
\hline Olr1256 & 6 & NM_001001086 & Selt & 1 & NM_001014253 \\
\hline Olr1257 & 10 & NM_001000596 & Selv & 1 & NM_001166396 \\
\hline Olr1273 & 6 & NM_001000458 & Sephs2 & 1 & NM_001079889 \\
\hline Olr1273 & 4 & NM_001000458 & Sepp1 & 10 & NM_0191921 \\
\hline Olr1274 & 2 & NM_001000801 & Sep15 & 1 & NM_001166396 \\
\hline Olr1275 & 4 & NM_001000800 & Sepw1 & 1 & NM_013027 \\
\hline Olr1475 & 2 & NM_001000027 & Slc21a4 & 7 & NM_030837 \\
\hline Olr19 & 9 & NM_001000117 & Snap25 & 1 & NM_001270576 \\
\hline Olr20 & 7 & NM_001000118 & Snx9 & 1 & NM_001127637 \\
\hline Olr440 & 6 & NM_001000282 & Stau2 & 2 & NM_001007149 \\
\hline Olr440 & 6 & NM_001000282 & Stxbp5l & 26 & NM_001271250 \\
\hline Olr5 & 2 & NM_001000112 & Taok1 & 9 & NM_173327 \\
\hline Olr520 & 1 & NM_001000930 & Trpm7 & 36 & NM_053705 \\
\hline Olr703 & 1 & NM_001000359 & Txnrd1 & 1 & NM_031641 \\
\hline Olr917 & 5 & NM_001001354 & Txnrd2 & 1 & NM_022584 \\
\hline Olr943 & 1 & NM_001001368 & Txnrd3 & 1 & NM_001184712 \\
\hline Plaur & 5 & NM_017350 & Usp3 & 3 & NM_001025424 \\
\hline Plb1 & 17 & NM_138898 & Vegfa & 1 & NM_001317043 \\
\hline Psmd10 & 4 & NM_053925 & Vimp & 1 & NM_173120 \\
\hline Ptpn18 & 4 & NM_001013111 & Vom2r73 & 1 & NM_001099486 \\
\hline Rabif & 12 & NM_001007678 & Zfp14 & 21 & NM_001100991 \\
\hline Rasgrf1 & 1 & NM_001105753 & & & \\
\hline Rbm25 & 4 & NM_001108984 & & & \\
\hline Reln & 1 & NM_080394 & & & \\
\hline RGD1305537 & 1 & NM_001108822 & & & \\
\hline
\end{tabular}




\section{Figure Legends}

Figure 1. Algorithm outputs and database design for CUT. Species specific inputs for yeast, rat and mouse genes are listed in the upper panel, with these gene (yeast) and transcript (rat and mouse) entries analyzed (middle panel) to catalog and provide searchable fields. Data provided in the CUT database includes species- and gene specific information on codon usage patterns and measures of the significance of said codon patterns in a specific gene (Z-score), relative to species specific genome measures.

Figure 2. CUT database overview. The design of the database utilized tables that track the codon sequence usage for each transcript and for each annotation set (genome).

Figure 3. Design features and data outputs using CUT. (A) Species specific data can be accessed and searched to provide data on specific genes, or identify genes with specific codon patterns ( 1 to 5 codons) and Z-scores, with a data search of the Budding Yeast gene YEF3 demonstrated. (B) Single codon patterns for the YEF3 highlight the number of highly used codons and their gene specific over usage (Z-score). For example in YEF3 the GAA codon is used 91 times and based on actual and expected frequencies a significant Z-score is observed. (C) As exampled using the YEF3 gene and the GAA-GAA dicodon that is found 8 times, CUT can be used to determine the number of times a dicodon is represented in a gene, with (D) all dicodons from a gene being able to be exported into a tab delimited file and analyzed to determine the most significant dicodon combinations. The top five most overused dicodons in YEF3 are represented on the graph, with the inset table detailing the next 25 .

Figure 4. Codon painting of specific genes. CUT can be used to identify codon patterns on a specific gene to identify the general position of specific codon runs in (A) ENT2, (C) NAB3 and (E) HMO1. AGA, GAA and AAG codons have all been linked to Trm9 regulated translation. Immunoblot analysis of the levels of specific 
proteins with (B) AGA-AGA for Ent2, (D) GAA-GAA-GAA-GAA-GAA for Nab3 and (F) AAG-AAG-AAG-AAGAAG for wild-type and trm9 $\Delta$ cells.

Figure 5. Dicodon trends in similarly regulated group of genes. All 64x64 dicodon patterns have been cataloged for each organism's genes/transcripts in CUT. Dicodon data on all species-containing genes can be downloaded and specific groups of genes can be analyzed for dicodon trends. Specifically, 80 transcripts that have been linked to regulation by Gcn4 were analyzed by summing each dicodon Z-score, with these values plotted for all 4,096 possible combinations. The AGA-GAA summed dicodon Z-score was determined to be significantly increased $\left(p<10^{-5}\right)$ for the Gcn4-regulated genes (inset panel). Significance was determined by random sampling of 80 transcripts 300 -times from the 6,664 yeast genes to determine the average summed Zscore and standard deviation for the AGA-GAA dicodon in a group. The summed Z-score for the 80 Gcn4regulated transcripts (68) was compared to the average score range of 80 random transcripts (28 to 33 ).

Figure 6. CUT tool organism downloads and dicodon data specific to mammalian genes. (A) Species specific information and gene specific monocodon and dicodon data for yeast, mouse and rat annotated genes and transcripts can be downloaded by clicking on the Download Stats Icon. Unique dicodon Z-scores indicating whether they are significantly over-used in mouse (B) Brca2 and (C) Gpx1. Note that the Y-scale is different when comparing Brca2 and Gpx1 data, as the latter translationally recodes UGA.

Figure 7. Translational recoding in mammals and CUT-based search for targets. In black, two potential mechanisms that can translate a UGA codon are detailed. In red, we describe the search space used to identify the 45 and 120 transcripts that contain internal UGA codons in mice and rats, respectively. 


\section{Yeast}

Saccharomyces cerevisiae

SGD_sacCer3

6,664 Total Genes

3,008,007 Total Codons

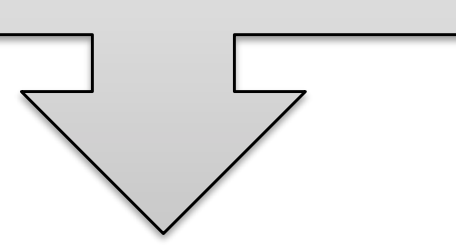

Rat

Rattus Norvegicus

RefSeq_rn5

16,711 Total Transcripts

$8,261,454$ Total Codons

\section{Mouse}

Mus Musculus

RefSeq_mm10

30,392 Total Transcripts

16,534,768 Total Codons

\section{Codon Utilization (CUT) Algorithm}

1. Catalogue gene specific codon combinations ( 1 to 5 codons) $X$ total transcripts. Possible - 64 singlet codons, 4096 double, 262,144 triple, 16,777,216 quadruple and $1,073,741,824$ quintuple codon combinations for each gene.

2. Determine genome average (expected) values for each codon combination.

3. Determine Z-scores for each gene specific codon combination (actual number expected) / Standard Deviation

\section{Codon Utilization (CUT) Database Outputs}

1. Searchable catalogue of all Saccharomyces cerevisiae and Mus Musculus genes to detail gene specific codon ( 1 to 5 ) patterns.

2. Genome based downloads of mono and dicodon frequencies and gene specific Zscores.

3. Genome specific searches for all genes with significant codon patterns based on codon pattern identity and/or Z-score.

4. Codon count, frequency calculations for input sequences, tabulating 1-5 codon combinations. 


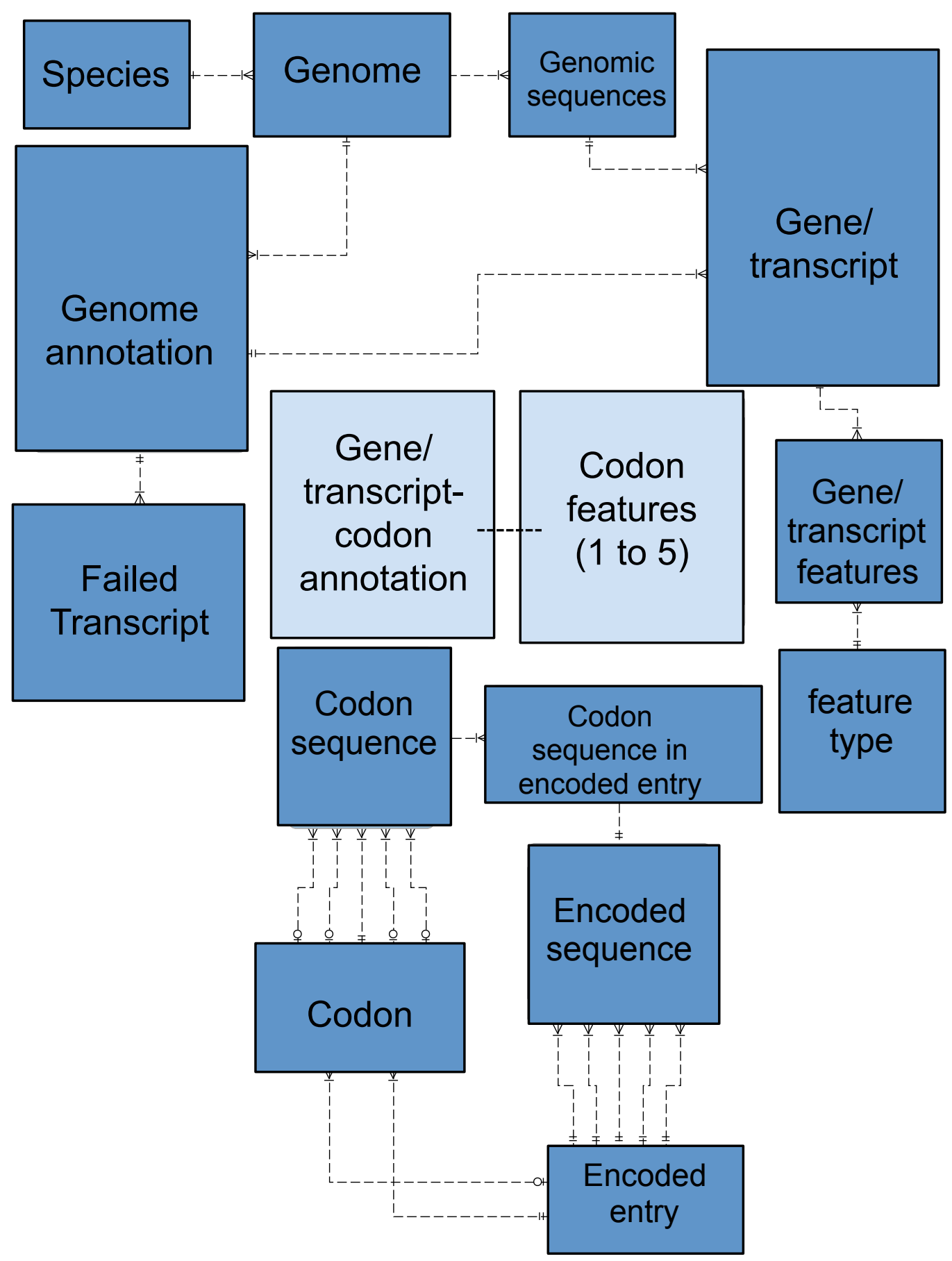




\section{A Codon Utilization Tool}

, Home, Search, View, Genomes , Analytics , Tutorial , Contact Info

Search genes:

\section{Annotation Filters:}

Organism: Yeast Genome: sacCer3

Annotation: SGD_sacCer3 $\neq$ Gene/Tx Name: yef3

CDS Filters:

Having any codon sequence with Z-Score greater than $+1-: 0.0$

Having any codon sequence of length 1 with Z-Score greater than $+1-: 0.0$

Having any codon sequence of length 2 with Z-Score greater than $+1=0.0$

Having any codon sequence of length 3 with Z-Score greater than $+1-: 0.0$

Having any codon sequence of length $\mathbf{4}$ with Z-Score greater than $+1-: 0.0$

Having any codon sequence of length 5 with Z-Score greater than $+1-: 0.0$

Containing codon sequence:

with Z-Score greater than $+1=0.0$

D

Search Genes

\section{C}

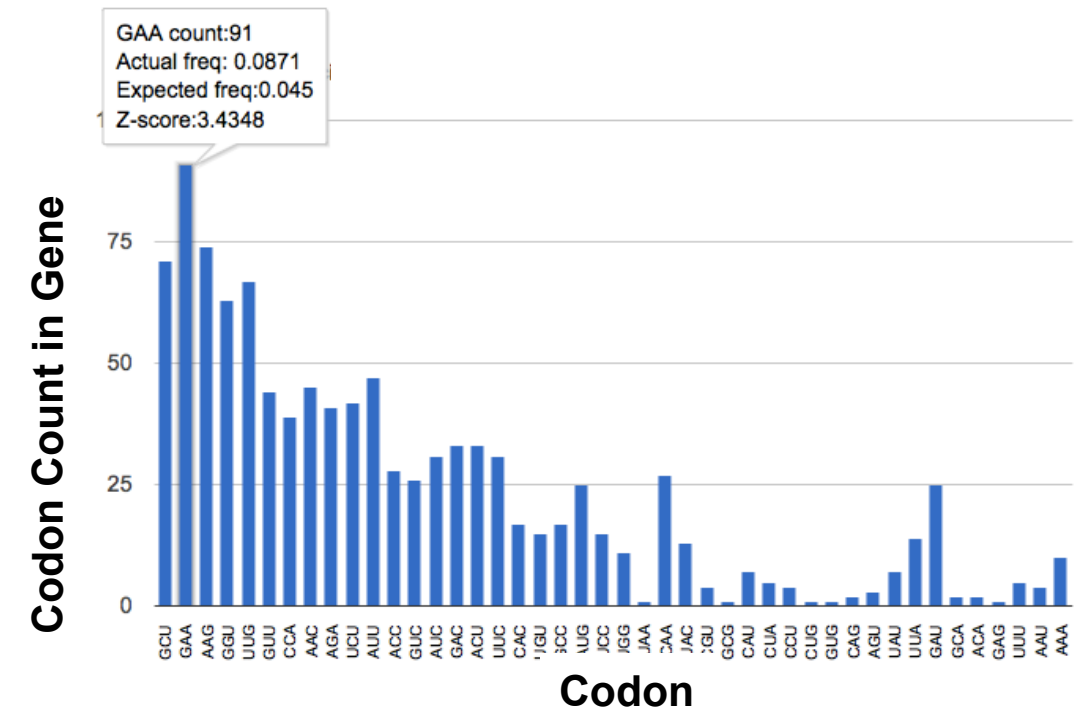

Codon Utilization Tool

, Home , Search , View , Genomes , Analyilics , Tutorial , Contact lifto

Gene CDS Viewer

Annotation Set Info:

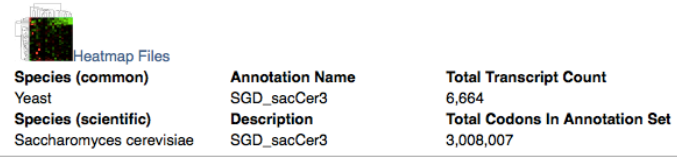

Transcript Into:

$$
\begin{array}{ll}
\text { Gene: YEF3 } & \text { Transcript: YLR229W } \\
\text { Codon count: 1,045 } & \text { Location: chrXIII(+):636780-639914 }
\end{array}
$$

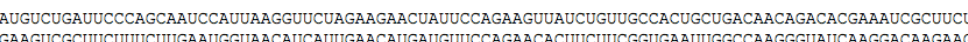

ACCGCUGCUAACGCCAUGCAAGCUGUUGCUCACAUUGCUAACCAAUCUAACUUGUCUCCAUCUGUUGAACCAUACAUCGUCCAAUUGGUUCCA

GCUAUCOGUAGCAACGCAGGUAACAAGGACAAAGAAAUUCAAUCUGUGGCUUCCGAAACUUUGAUUUCCAUCGUUAACGCUGUUAACCCAGU

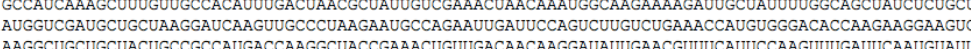

AAGGCUGCUGCUACUGCCGCCAUGACCAAGGCUACCGAAACUGUUGACAACAAGGAUAUUGAACGUUUCAUUCCAAGUUUGAUUCAAUGUAUU
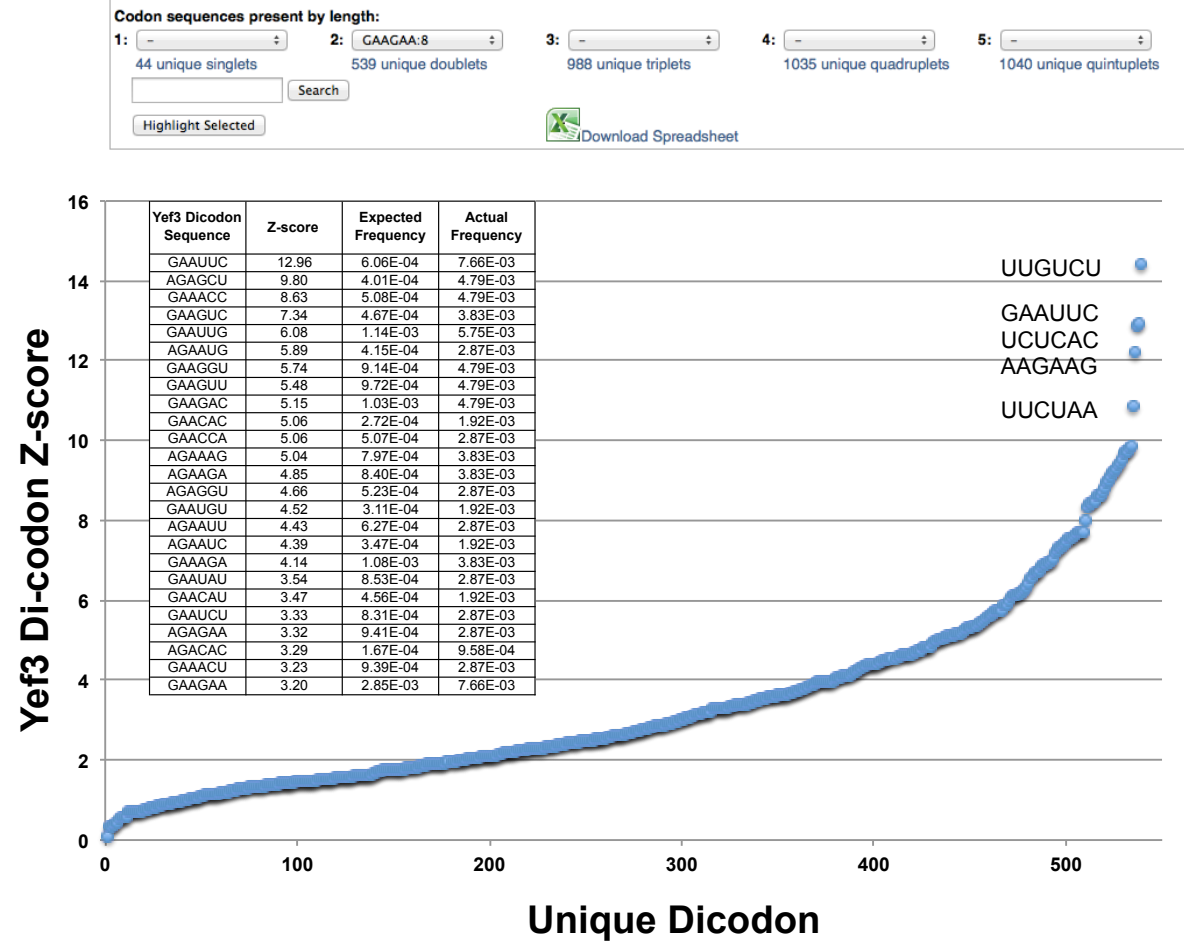
A
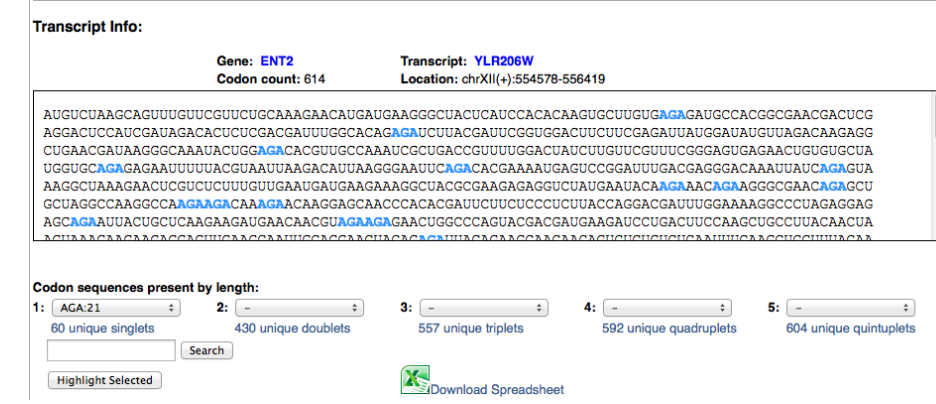

B
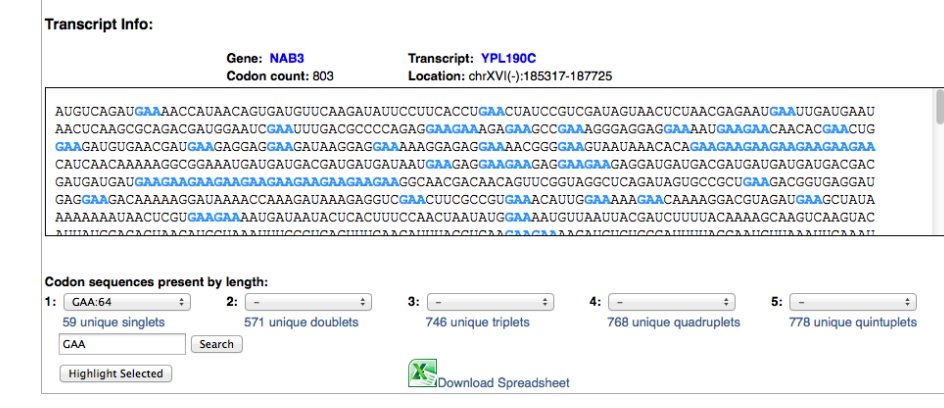

E
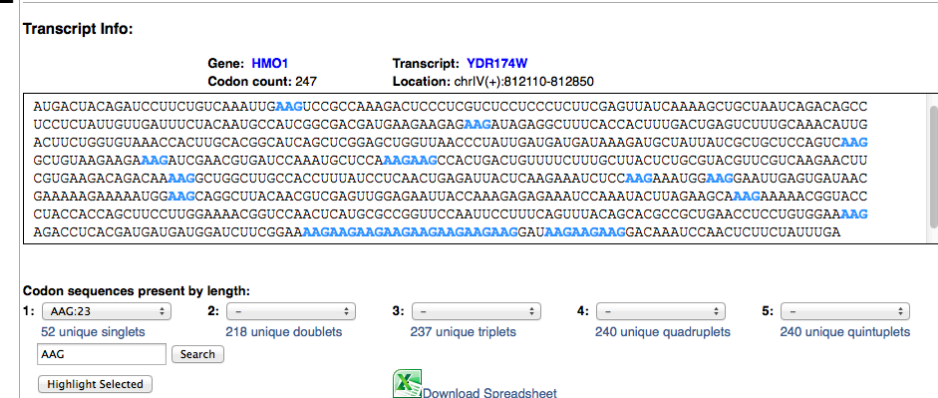

C

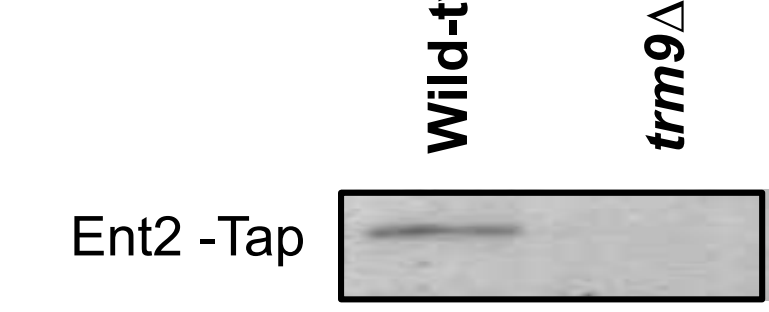

D

Tubulin

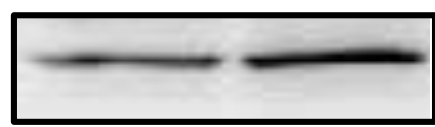

AGAAGA

Count $=2$

Actual freq. $=\mathbf{0 . 0 0 2 1}$

Expect. Freq. $=0.009$

Z-score $=2.03$

\section{Nab3 -Tap

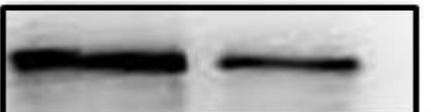 \\ GAAGAAGAAGAAGAA \\ Count $=9$}

Tubulin

$\mathbf{F}$

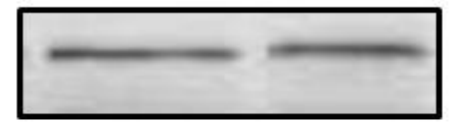

Hmo1 -Tap

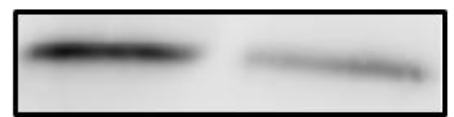

Tubulin
Actual freq. $=0.0013$

Expect. Freq. $=0$

Z-score $=27.88$
AAGAAGAAGAAGAAG Count $=4$

Actual freq. $=0.0165$

Expect. Freq. $=0$

Z-score $=55.19$ 


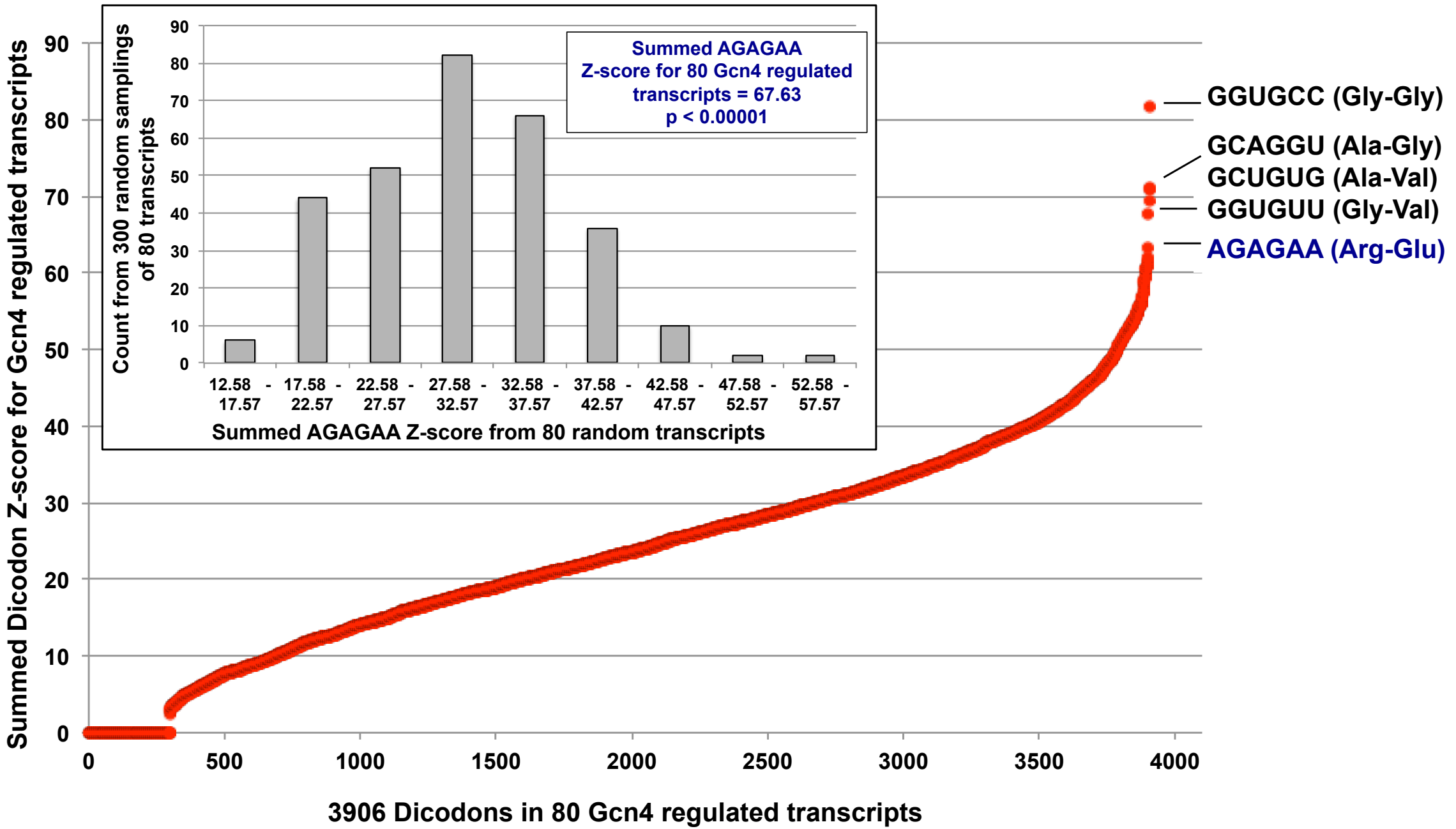




\section{A Genome Annotations in CUT}

\section{Species}

Yeast(Saccharomyces cerevisiae)

Mouse(Mus Musculus)

Rat(Rattus Norvegicus)
Annotation Name

$\begin{array}{lll}\text { SGD_sacCer3 } & 6,664 & 3,008,007 \\ \text { RefSeq_mm10 } & 30,392 & 16,534,768 \\ \text { RefSeq_rn5 } & 16,711 & 8,261,454\end{array}$

Genomic Codon Usage Statistics

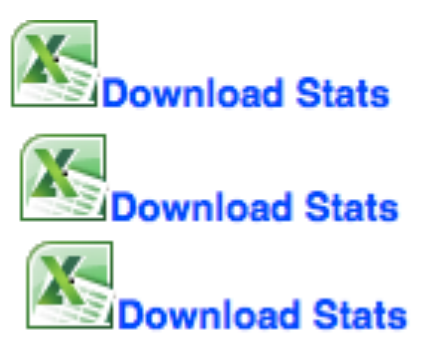

C

B

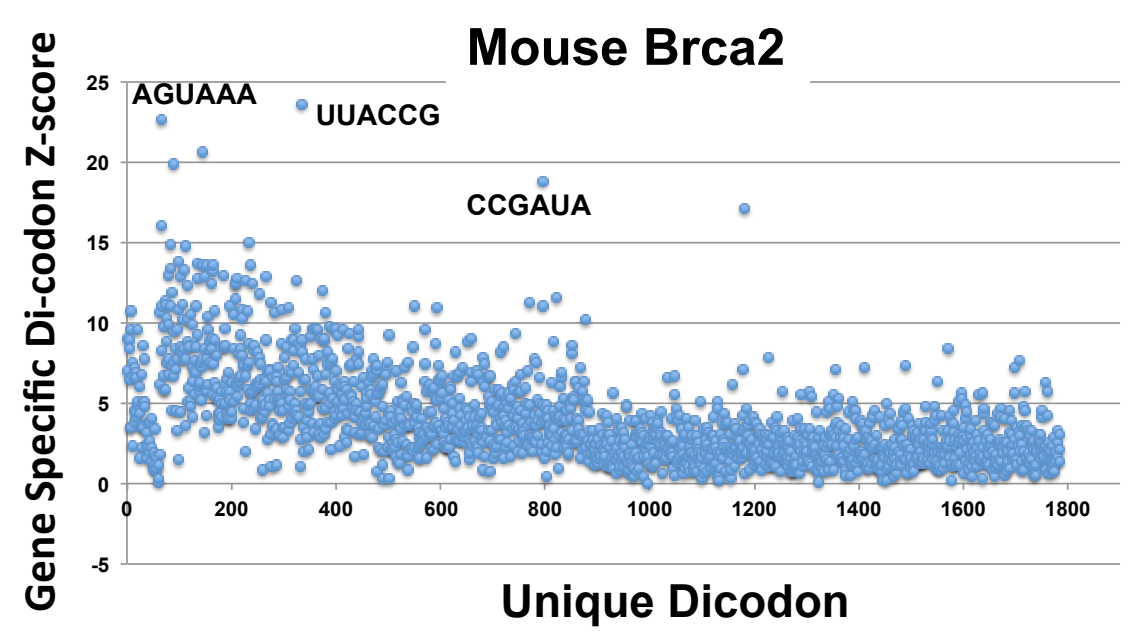

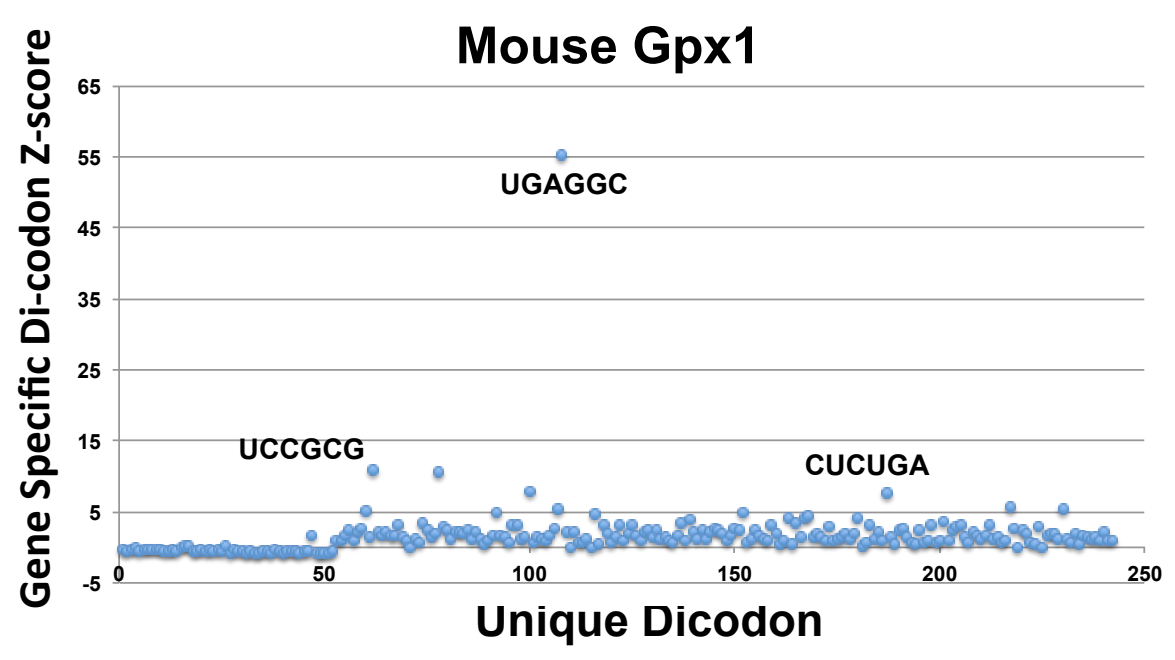




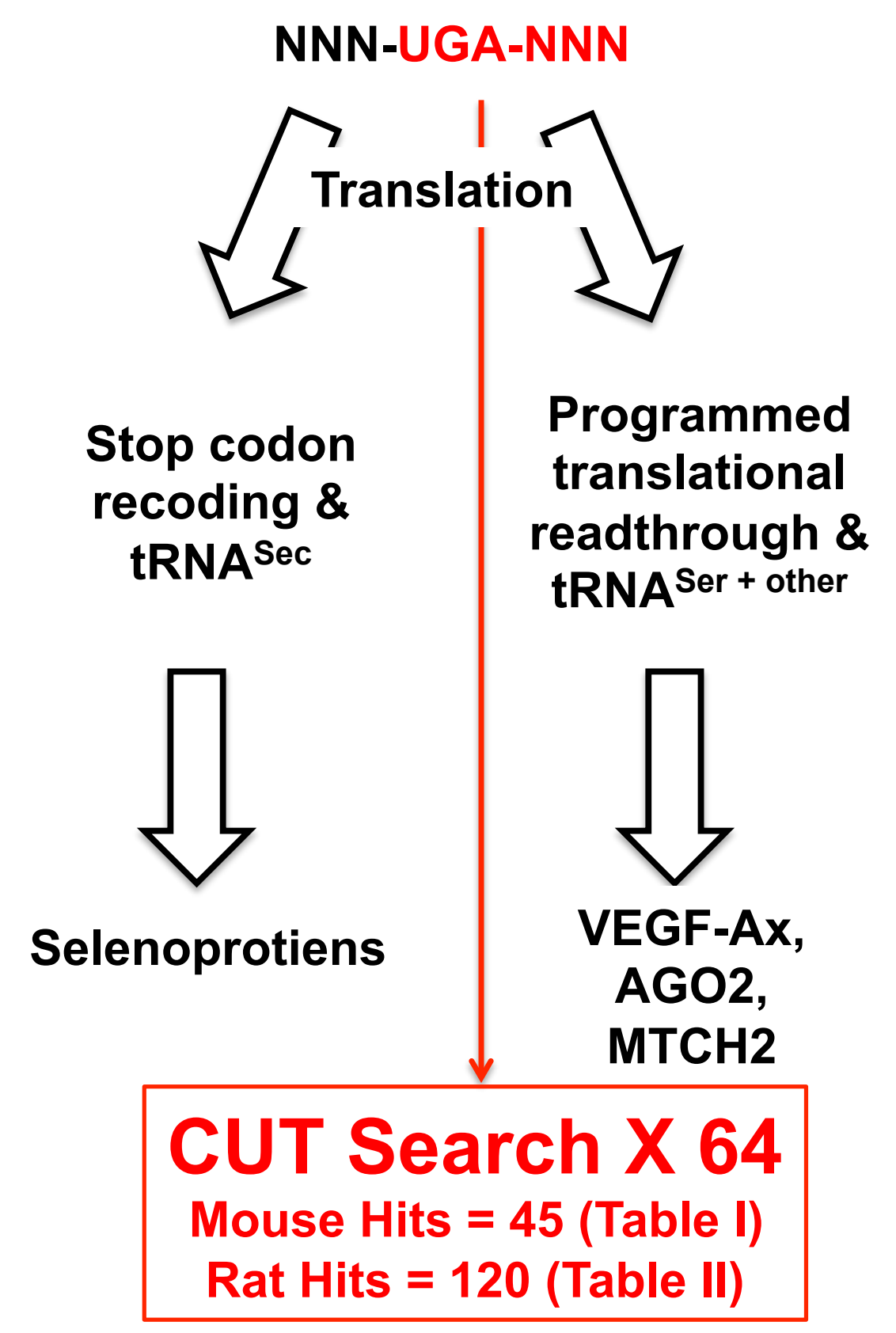

Stop codon tRNA ${ }^{\mathrm{Sec}}$

Selenoprotiens

VEGF-Ax, AGO2, $\mathrm{MTCH} 2$

\section{CUT Search X 64 \\ Mouse Hits = 45 (Table I) Rat Hits = 120 (Table II)} translational
readthrough \&
tRNA translational
readthrough \&
tRNA

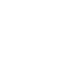

NNN-UGA-NNN

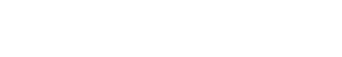

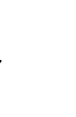

